\title{
JMSR
}

Journal of Medical and Scientific Research

\section{Primary advanced non-Hodgkin's lymphoma of bladder with bilateral hydroureteronephrosis: A case report}

\author{
Gupta Aditya $\mathbf{M}^{1,}$, Shetty Raisa $\mathbf{N}^{1}$, and Amlani Pratik ${ }^{1}$ \\ ${ }^{1}$ Department of Urology,Yashoda hospital, Secunderabad, Telangana-500003, India
}

\begin{abstract}
Primary lymphoma of bladder is a very rare entity with a $0.2 \%$ incidence of all bladder malignancies. Secondary bladder involvement in malignant lymphoma is more common (10-25\%) with preponderance in middle aged females. A non-Hodgkin lymphoma of the bladder can be managed conservatively without surgery and has a better prognosis. Because the presenting symptoms and radiological imaging cannot conclusively rule out a primary bladder lymphoma from other malignant causes, cystoscopy and biopsy with immune-histochemical staining is essential. We present to you a 72-year-old male with complaints of irritative lower urinary tract symptoms and asymptomatic microhematuria. On evaluation computerized tomography (CT) was s/o a large bladder wall mass with bilateral mild hydroureteronephrosis and enlarged pelvic lymphnodes. Cystoscopy and biopsy was done and the report came out to be a non-Hodgkins lymphoma. Whole body positron emission tomography (PET) scan was done which confirmed it to be a primary bladder NHL. Patient was treated with chemotherapy and is now doing well on follow up. We would like to share our experience in managing this case.
\end{abstract}

Keywords: non-Hodgkin lymphoma; bladder malignancy; cystoscopy

*Corresponding Author: Dr. Aditya Gupta, 1-c, Sabarmati Industrial Society near Indus Hospital, Sabarmati, Ahmedabad, Gujarat 380005. Mobile: 9979971024; Email: dradityauro@ gmail.com

Received 5 April 2021; Revised 12 June 2021; Accepted 22 June 2021; Published 2 July 2021

Citation: Aditya MG, Raisa NS, Pratik A. Primary advanced non-Hodgkin's lymphoma of bladder with bilateral hydroureteronephrosis: A case report. J Med Sci Res. 2021; 9(3):1184-186. DOI: http://dx.doi.org/10.17727/JMSR.2021/927

Copyright: (C) 2021 Aditya MG et al. Published by KIMS Foundation and Research Center. This is an open-access article distributed under the terms of the Creative Commons Attribution License, which permits unrestricted use, distribution, and reproduction in any medium, provided the original author and source are credited.

\section{Introduction}

Bladder malignancies account for almost $3.5 \%$ of the total cancer burden world-wide. Most of them arise from the epithelium and in general does not have as good a prognosis as one would hope for. In such a scenario, encountering a cancer such as primary bladder non-Hodgkin lymphoma is a sigh of relief. Primary lymphoma of bladder is a very rare entity with a $0.2 \%$ incidence of all bladder malignancies [1]. Most of the bladder involvement in lymphoma's is secondary (10-25\%) with preponderance in middle aged females [2]. Primary lymphoma of bladder are most commonly mucosa-associated lymphoid tissue (MALT) lymphoma and diffuse large B-cell lymphoma (DLBCL) type. Mode of presentation is similar to the common bladder malignancies: hematuria, dysuria, 
frequency, supra pubic pain. Complications like fistula, full bladder involvement, hydronephrosis secondary to ureteral obstruction are very rare. It is very important to differentiate a primary lymphoma of bladder from other routine bladder malignancies because of the substantial treatment difference. Because the presenting symptoms and radiological imaging cannot conclusively rule out a primary bladder lymphoma from other malignant causes, biopsy with IHC staining is required. ${ }^{18} \mathrm{~F}$ fluorodeoxyglucose-positron emission tomography (FDG-PET/CT) plays a central role to rule out concomitant systemic disease. Even a high grade non-Hodgkin lymphoma of the bladder can be managed successfully with chemotherapy.

\section{Case report}

A72-year-old male came with complaints of increased urinary frequency and urgency for 6 months. There were no voiding symptoms or systemic complaints. Routine blood investigations were normal but urine $\mathrm{r} / \mathrm{m}$ showed microhematuria. Ultrasound bladder showed mild bilateral hydroureteronephrosis with bladder thickening. Contrast enhanced CT scan (abdomen and pelvis) was done which was s/o a $9 \times 7 \mathrm{~cm}$ isodense bladder wall mass predominantly on the left side infiltrating b/l distal ureters and enlarged pelvic lymph nodes (Figure 1). Labelling it as advanced bladder malignancy, cystoscopy with TURB was planned.

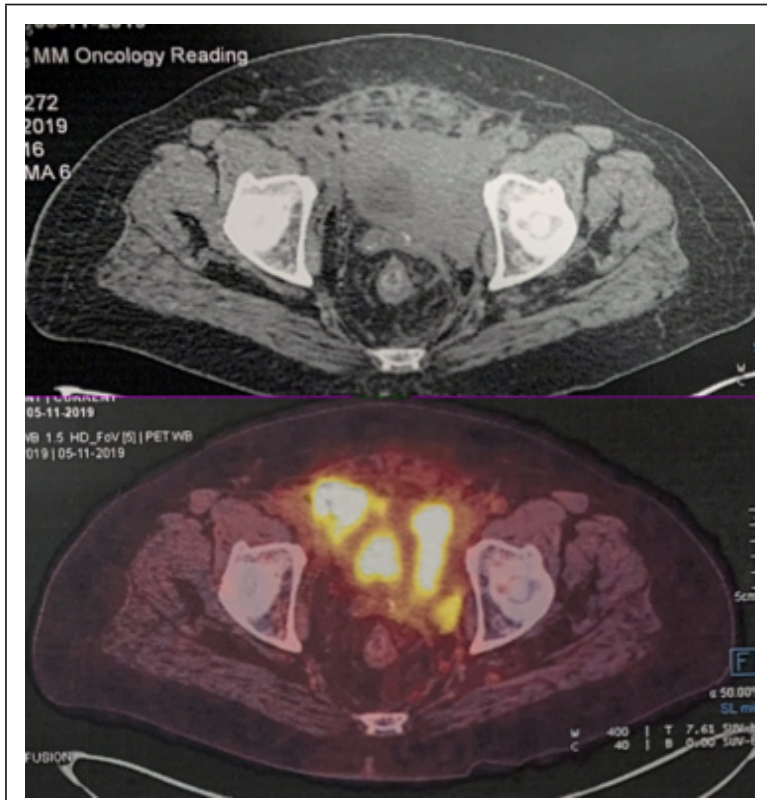

Figure 1a, b: Left bladder mass infiltrating bilateral distal ureters and enlarged pelvic nodes.
On cystoscopy a large smooth, non-ulcerative submucosal mass was found predominantly at posterior wall with failure to visualise left ureteral orifice. Biopsy was taken and patient was planned for neoadjuvant chemotherapy. However biopsy was suggestive of atypical lymphoid infiltration and on IHC, cells were diffusely positive for CD20 and was negative for $\mathrm{CD} 3,10,34,68$ and Bcl2 which confirmed non-Hodgkin's lymphoma. FDG PET/CT was done which ruled out systemic disease and finally the diagnosis of Primary Malignant NHL of bladder was kept (Figure 2).

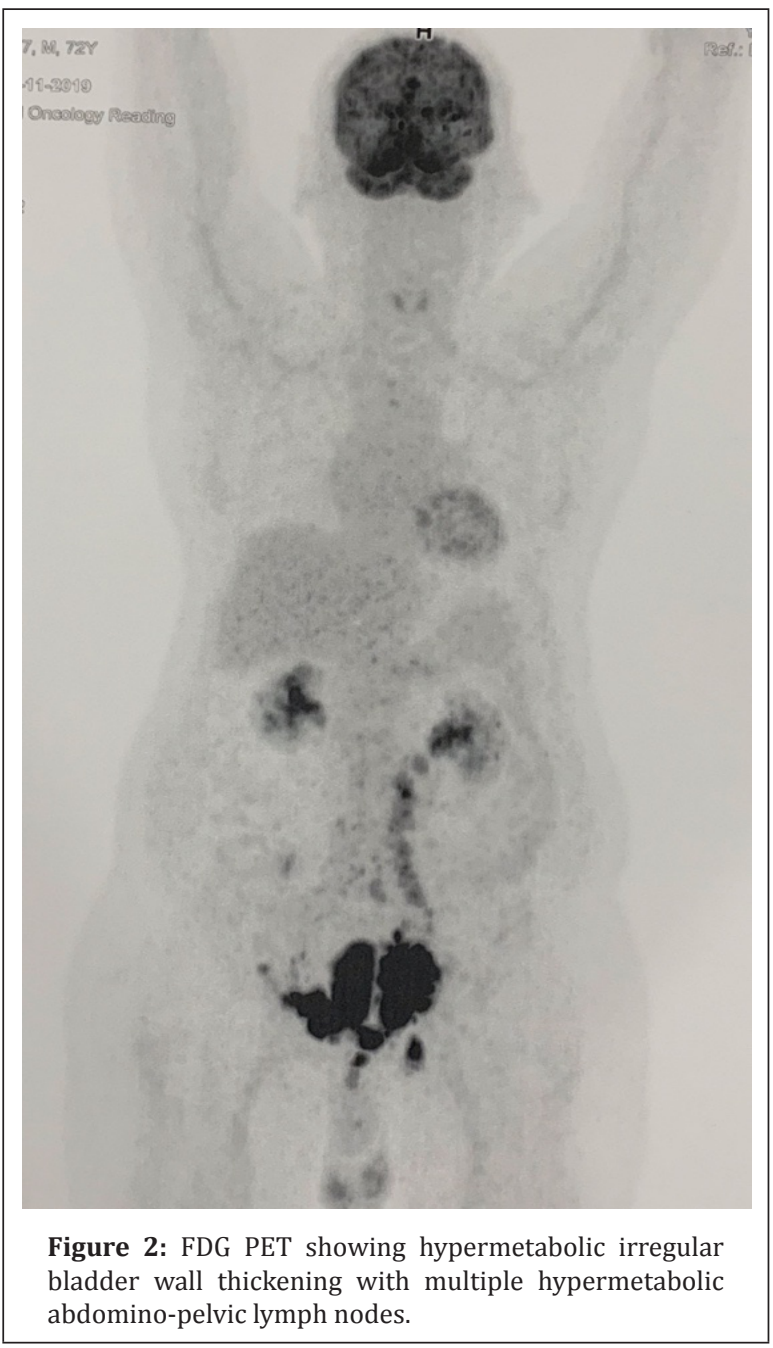

Patient was given 6 cycles of R-CHOP (Rituximab, cyclophosphamide, vincristine, doxorubicin, prednisolone). Patient tolerated the treatment well with mild side effects. A repeat PET scan and cystoscopy was done at 3 months which was found to be normal. The patient is now on follow up and doing well. 


\section{Discussion and conclusion}

Primary lymphoma originating in the urinary bladder is exceedingly rare. A possible explanation is that as there are no germinal follicles or lymphoid tissue developing in the bladder itself, these lymphomas should develop in the background of a preexisting cystitis; $22-40 \%$ of the reported cases were preceded by chronic cystitis [3]. Differentiating from routine bladder malignancies is difficult as the presentation and radiological features are almost similar in both. Lymphoma of the bladder is proposed to have characteristic cystoscopic appearance that can aid in diagnosis and is usually descried as a smooth, nonulcerative, friable or hemorrhagic submucosal tumor [4]. Histology with IHC generally clinches the diagnosis. FDG PET/CT should be done to look for systemic involvement particularly in high grade lymphomas. For treatment: chemotherapy, radiotherapy, surgery or their combination are the options available. Chemotherapy with a R-CHOP (cyclophosphamide/ doxorubicin/ vincristine/ prednisolone) regimen is used most frequently [5] and most of the times is associated with a complete response. Thus though this patient had high risk features for routine bladder malignancy (b/l Hdun, large bladder mass, enlarged pelvic lymph nodes) which would require neoadjuvant therapy with radical cystectomy, he was successfully managed with just chemotherapy as it was a primary NHL of bladder.

\section{Conflicts of interest}

Authors declare no conflicts of interest.

\section{References}

[1] Kuhara H, Tamura Z, Suchi T, Hattori R, Kinukawa T. Primary malignant lymphoma of the urinary bladder: A case report. Acta Pathol Jpn. 1990; 40(10):764-769.

[2] Sufrin G, Keogh B, Moore RH, Murphy GP. Secondary involvement of the bladder in malignant lymphoma. J Urol. 1977; 118(2):251-253.

[3] Bates AW, Norton AJ, Baithun SI. Malignant lymphoma of the urinary bladder: A clinicopathological study of 11 cases. J Clin Pathol. 2000; 53(6):458-461.

[4] Downs TM, Kibel AS, DeWoif WC. Primary lymphoma of the bladder: a unique cystoscopic appearance. Urology. 1997; 49(2):276-278.

[5] Oh KC, Zang DY. Primary non-Hodgkin's lymphoma of the bladder with bone marrow involvement. Korean J Intern Med. 2003; 18(1):40-44. 